\title{
DIFERENCIAS DE EDAD Y GÉNERO EN COMPORTAMIENTO SOCIAL, TEMPERAMENTO Y REGULACIÓN EMOCIONAL EN NIÑOS ARGENTINOS
}

\author{
Cecilia Reyna*, Silvina Brussino \\ Centro de Investigaciones de la Facultad de Psicología (CIPSI), Grupo Vinculado al Centro de Investigaciones y Estudios sobre Cultura \\ y Sociedad (CIECS), Consejo Nacional de Investigaciones Científicas y Técnicas (CONICET) - Universidad Nacional de Córdoba, Argentina \\ Recibido, septiembre 14/2013 \\ Concepto de evaluación, mayo 5/2014 \\ Aceptado, mayo 26/2015 \\ Referencia: Reyna, C. \& Brussino, S. (2015). Diferencias \\ de edad y género en comportamiento social, temperamento y \\ regulación emocional en niños argentinos. Acta Colombiana de \\ Psicología, 18 (2), 51-64. DOI: 10.14718/ACP.2015.18.2.5
}

Resumen

El objetivo de esta investigación fue evaluar las diferencias en función de la edad, el género y el nivel socio-económico en el comportamiento social (habilidades sociales y problemas de conducta), la atención focalizada, el control inhibitorio, la emocionalidad (positiva y negativa) y la regulación emocional (positiva y negativa) en niños argentinos de tres, cinco y siete años de edad. Se realizó un estudio evolutivo transversal en el cual participaron los padres y docentes de 623 niños. Los padres brindaron información acerca de aspectos socio-demográficos, y sobre el temperamento y procesos emocionales de los niños, mientras que los docentes lo hicieron con respecto al comportamiento social de los niños. El estudio de las comparaciones entre grupos se efectuó a través de análisis de varianza. Las diferencias más marcadas se atribuyeron, en primer lugar, a la edad de los niños, y en segundo lugar, al género. En términos generales, los niños de tres años mostraron un menor desempeño que el resto de los grupos, pero el desarrollo no fue lineal en todos los procesos ya que los niños de cinco años presentaron un desempeño semejante o mejor en comparación con los niños de siete años, según algunas variables.

Palabras clave: temperamento, emociones, habilidades sociales, problemas de conducta, niños.

\section{AGE AND GENDER DIFFERENCES IN SOCIAL BEHAVIOR, TEMPERAMENT AND EMOTIONAL REGULATION AMONG ARGENTINEAN CHILDREN}

\begin{abstract}
The purpose of this study was to assess the differences in terms of age, gender and socioeconomic background in social behavior (social skills and behavior problems), focused attention, inhibitory control, positive and negative emotionality and regulation of positive and negative emotions in Argentinean children aged 3, 5 and 7 years. A developmental cross-sectional study was carried out where the parents and teachers of 623 children participated. Parents reported on socio-demographic issues, children's temperament and emotional processes, while teachers provided information on children's social behavior. Comparisons between groups were conducted by analysis of variance. The most marked differences were attributed, firstly, to children's age, and secondly, to gender. Overall, the children aged three showed a lower performance than the other groups, but development was not linear on all the processes, as the children aged five showed a similar or better performance than the children aged seven when considering several variables.

Key words: temperament, emotions, social skills, behavioral problems, children.

\section{DIFERENÇAS DE IDADE E GENERO EM COMPORTAMENTO SOCIAL, TEMPERAMENTO E REGULAÇÁOO EMOCIONAL EM CRIANÇAS ARGENTINAS}

Resumo

\begin{abstract}
O objetivo desta pesquisa foi avaliar as diferenças em função da idade, do gênero e do nível socioeconômico no comportamento social (habilidades sociais e problemas de conduta), a atenção focalizada, o controle inibitório, a emocionalidade (positiva e negativa) e a regulação emocional (positiva e negativa) em crianças argentinas de três, cinco e sete anos de idade. Realizou-se um estudo evolutivo transversal no qual participaram os pais e docentes de 623 crianças. Os pais deram informação sobre os
\end{abstract}

\footnotetext{
*ecilia Reyna, Enrique Barros y Enfermera Gordillo, Facultad de Psicología, Ciudad Universitaria, CP: 5000, Córdoba, Argentina. +54 3514344984 int. 149. ceciliareyna@gmail.com
} 


\begin{abstract}
aspectos sócio demográficos, e sobre o temperamento e processos emocionais das crianças, enquanto os docentes o fizeram com respeito ao comportamento social das crianças. O estudo das comparações entre grupos efetuou-se através de uma análise de variância. As diferenças mais marcadas atribuíram-se, em primeiro lugar, à idade das crianças, e em segundo lugar, ao gênero. Em termos gerais, as crianças de três anos mostraram um desempenho pior que o resto dos grupos, mas o desenvolvimento não foi linear em todos os processos já que as crianças de cinco anos apresentaram um desempenho semelhante ou melhor em comparação com as crianças de sete anos, segundo algumas variáveis.

Palavras chave: temperamento, emociones, habilidades sociais, problemas de conduta, crianças.
\end{abstract}

\section{INTRODUCCIÓN}

El objetivo de este estudio fue evaluar las diferencias en función de la edad, el género y el nivel socio-económico en el comportamiento social (habilidades sociales y problemas de conducta), la atención focalizada, el control inhibitorio, la emocionalidad (positiva y negativa) y la regulación emocional (positiva y negativa) en niños argentinos de tres, cinco y siete años de edad. A continuación se revisan aspectos teóricos y antecedentes específicos sobre las diferencias de interés en cada uno de los constructos, exponiéndose oportunamente las hipótesis de trabajo. Luego se exponen aspectos metodológicos, y se presentan los resultados. En el último apartado se discuten los mismos a la luz de las teorías y antecedentes más relevantes.

\section{Comportamiento social}

Se entiende por competencia social la efectividad en la interacción, lo cual implica el desarrollo de conductas organizadas que se evidencian en distintos momentos de la vida (Rose-Krasnor, 1997) y que son percibidas como positivas por personas del entorno (Gresham, 2001). Una de las formas de concebir la competencia social es a partir de componentes, lo que ha dado origen a los conceptos de habilidades sociales y comportamientos sociales específicos que se manifiestan en actividades como iniciar una conversación, jugar con pares o agradecer (Gresham, Sugai \& Horner, 2001), y que constituyen la competencia social relacionada con estructuras motivacionales y afectivas (Trianes, Muñoz \& Jiménez, 1997). Ambos conceptos se enmarcan dentro del comportamiento adaptativo. Para que los niños logren un desarrollo social adecuado no solo deben adquirir habilidades sociales para interactuar con otros, sino que deben ser capaces de utilizar esas habilidades de manera aceptable para su entorno (Sheridan \& Walker, 1999).

La contra cara del comportamiento adaptativo son los problemas de conducta. En un estudio sobre la clasificación de síntomas patológicos en la infancia, Achenbach (1966) identificó dos amplias categorías: problemas externalizantes y problemas internalizantes, categorías que se utilizan en la actualidad. Los problemas externalizantes se refieren a acciones hacia el exterior y comprenden comportamientos no controlados, acting-out y agresión, mientras que los problemas internalizantes hacen referencia, principalmente, a problemas tales como ansiedad, depresión, aislamiento social y quejas somáticas (Cicchetti \& Toth, 1991; Sewrud-Clikeman, 2007).

\section{Rol de la edad en el comportamiento social}

El avance de los años va acompañado por un creciente desarrollo de la competencia social y la disminución de los problemas externalizantes debido a la acumulación de experiencias de interacción y desarrollo del lenguaje, la capacidad cognitiva y la regulación emocional (Hill, Degnan, Calkins \& Keane, 2006; Miner \& Clarke-Stewart, 2008; Thompson \& Goodvin, 2007); en tanto que se espera que los problemas internalizantes se incrementen gradualmente producto de la maduración cognitiva (Kovacs \& Devline, 1998). Sin embargo, esas suposiciones no son determinantes a la hora de caracterizar el curso de desarrollo del comportamiento social, lo cual se atribuye, al menos en parte, a las demandas cambiantes y expectativas diferenciales en tempranas etapas evolutivas (Keenan \& Shaw, 1997). Si bien algunos autores han observado perfiles de desarrollo particulares de distintos tipos de conductas prosociales (p.e., Persson, 2005), se espera un incremento semejante para las distintas dimensiones de las habilidades sociales.

Hipótesis 1. Las habilidades sociales (puntuación total y dimensiones) se incrementan con la edad; los niños de tres años presentan menos habilidades sociales que los niños de cinco años, y estos, a la vez, menos habilidades sociales que los niños de siete años.

Hipótesis 2. Los problemas de conducta en general y los problemas externalizantes disminuyen con los años; los niños de tres años presentan más problemas de conducta y problemas externalizantes que los niños de cinco años, quienes manifiestan más problemas de conducta y problemas externalizantes que los niños de siete años.

Hipótesis 3. Los problemas internalizantes se incrementan con los años: los niños de tres años muestran menos problemas internalizantes que los niños de cinco años, y estos, a la vez, menos problemas internalizantes que los de siete años. 


\section{Rol del género en el comportamiento social}

La mayoría de los estudios sobre desarrollo social infantil señalan que las niñas presentan mayores habilidades sociales, y que los varones se caracterizan por más problemas de conducta externalizante (Connor, 2002). En cuanto a los problemas internalizantes, aunque existe alguna evidencia sobre mayores niveles en niñas durante los años escolares y en la adolescencia inicial y media (p.e., Keiley et al., 2003), los hallazgos en etapas tempranas son escasos. Si bien algunos estudios señalan diferencias específicas de género según distintos tipos de comportamiento agresivo (Ostrov \& Keating, 2004) y en habilidades sociales particulares (Souza \& Rodrigues, 2002), se espera que las diferencias en las dimensiones de habilidades sociales y problemas de conducta consideradas en este estudio se manifiesten en la misma dirección que las correspondientes escalas globales. Por otra parte, teniendo en cuenta la ausencia de diferencias de género reportada por algunos investigadores en edades tempranas (p.e., Teague, 2005), brecha que se supone se incrementa a partir de los cuatro años, solo se espera encontrar diferencias en el comportamiento social según el género en los niños de cinco y siete años (efecto de interacción).

Hipótesis 4. Las niñas presentan mayores habilidades sociales (puntuación total y dimensiones) que los varones. Además, se hipotetiza un efecto de interacción: las niñas de cinco y siete años presentan mayores habilidades sociales (puntuación total y dimensiones) que los varones de cinco y siete años, respectivamente; en cambio, los niños de tres años no se diferencian entre sí en habilidades sociales.

Hipótesis 5. Los varones presentan mayores problemas de conducta (puntuación total y dimensiones) que las niñas. Asimismo, se hipotetiza un efecto de interacción: los varones de cinco y siete años presentan mayores problemas de conducta (puntuación total y dimensiones) que las niñas de cinco y siete, respectivamente; por el contrario, los niños de tres años no se diferencian entre sí en los problemas de conducta según el género.

\section{Rol del nivel socio-económico en el comportamiento social}

Diversos estudios han señalado que el nivel socioeconómico (NSE) impacta en el funcionamiento cognitivo y socio-emocional del niño (para una revisión ver Bradley $\&$ Corwyn, 2002).Específicamente, se ha resaltado el impacto negativo de la pobreza sobre el desarrollo infantil (Grantham-McGregor et al., 2007). Como señalan BrooksGunn y Duncan (1997), los niños que viven en condiciones de pobreza son más susceptibles de evidenciar problemas comportamentales y emocionales, aunque ello no se generaliza a todos los niños que viven en dichas condiciones. En este estudio se espera ver reflejado, a través del NSE, el impacto negativo de malas condiciones socio-económicas sobre el comportamiento social infantil (Bandeira et al., 2006; Propper \& Rigg, 2007).

Hipótesis 6. Los niños caracterizados por NSE bajo resultan menos habilidosos en términos sociales que los niños con mayor NSE, con menos habilidades sociales y más problemas de conducta (puntuaciones globales y dimensiones).

\section{Temperamento y regulación emocional}

En la actualidad no hay una definición unívoca de temperamento, aunque el modelo más influyente es el de Mary Rothbart. Desde esta concepción se define el temperamento como las diferencias individuales en la reactividad y autorregulación que tienen base constitucional, y se manifiestan en el dominio de la emoción, actividad y atención (Rothbart, 1981; Rothbart \& Bates, 1998). Rothbart, Ahadi, Hershey, y Fisher (2001) han identificado tres grandes factores del temperamento infantil: afectividad negativa, saliencia-extraversión, y control voluntario o con esfuerzo. La emocionalidad ocupa un rol central, y es definida como diferencias individuales en los límites de reacción, latencia, intensidad y tiempo de recuperación, es decir, cuán fácil y cuán intensamente las emociones son activadas (Eisenberg et al., 1996). El factor saliencia-extraversión es una amplia categoría que comprende, entre otros aspectos, la emocionalidad positiva/exuberancia, que se caracteriza por risas y sonrisas, placer, alegría y extraversión (Gartstein \& Rothbart, 2003; Shiner \& Caspi, 2003).

Entre los aspectos "fríos" del temperamento se destacan dos procesos enmarcados en el control voluntario. Por una parte, el cambio o focalización de la atención, proceso que permite modular la activación emocional, regulando la exposición a un estímulo y los procesos cognitivos relacionados con esas experiencias emocionales (Rothbart, Ahadi \& Evans, 2000), permitiendo de esa manera manejar las conductas manifiestas que se asocian con las emociones (Eisenberg, Fabes, Guthrie \& Reiser, 2000). Por otra parte, el control inhibitorio, el cual se refiere a la capacidad para planificar y suprimir respuestas de aproximación inadecuadas (Rothbart et al., 2000).

Con respecto a la regulación emocional, si bien es un concepto extensamente discutido (Bridges, Denham \& Ganiban, 2004; Cole, Martin \& Dennis, 2004; Eisenberg \& Spinrad, 2004), se la concibe como un conjunto de procesos intrínsecos y extrínsecos que permiten monitorear, evaluary modular reacciones emocionales con el fin de lograr metas sociales, comportamentales y/o emocionales particulares (Eisenberg, Fabes \& Guthrie, 1997), como un proceso que puede ocurrir a nivel consciente o no, e implicar alguna 
modificación, ya sea de la propia respuesta o de la situación que genera la emoción (Diamond \& Aspinwall, 2003).

\section{Rol de la edad en la atención focalizada y el control in- hibitorio}

Los rasgos temperamentales muestran una estabilidad moderada a lo largo del tiempo, con posibles cambios producto de factores ambientales y de la activación/desactivación de sistemas genéticos en distintos períodos del desarrollo (Gottlieb, 2003; Rothbart, Ahadi, et al., 2001). Resultados particulares se observan al considerar las distintas dimensiones del temperamento. El período preescolar resulta clave para el control inhibitorio y la atención focalizada; el progreso del control ejecutivo de la atención como consecuencia de la maduración cerebral y la interacción con el ambiente favorece el desarrollo de habilidades autorregulatorias más sofisticadas, como la planificación de la conducta, la inhibición de respuestas inapropiadas y la focalización y el cambio de la atención (Kochanska, Murray \& Harlan, 2000; Rothbart, Chew \& Gartstein, 2001).

Hipótesis 7. Las capacidades de atención focalizada y el control inhibitorio se incrementan con el pasar de los años; los niños de tres años presentan menos habilidades que los de cinco años, y estos, menos que los de siete años.

\section{Rol de la edad en la emocionalidad}

En cuanto a las emociones, a lo largo de los años se aprecia diversificación, surgimiento de nuevas emociones y disminución de la frecuencia e intensidad de la expresión emocional, tornándose cada vez más propensas a la regulación y control voluntario (Holodynski \& Friedlmeier, 2006). $\mathrm{Si}$ bien en los primeros dos años se aprecia un incremento global en emocionalidad, posteriormente los resultados son diferentes. En cuanto a emocionalidad negativa, Murphy, Eisenberg, Fabes, Shepard y Guthrie (1999) sugieren una disminución a lo largo de los años luego de un pico a los tres años. Por el contrario, respecto de la emocionalidad positiva/exuberancia, hay estudios que señalan un incremento de los tres años y medio a los cinco años, para luego permanecer en niveles estables (Guerin \& Gottfried, 1994).

Hipótesis 8. La emocionalidad negativa disminuye con el avance de los años en el período evolutivo comprendido; los niños de tres años presentan niveles mayores que los de cinco años, y estos, mayores que los de siete años.

Hipótesis 9. La emocionalidad positiva/exuberancia se incrementa hasta los cinco años y luego se mantiene estable; los niños de tres años manifiestan menos emocionalidad positiva que los niños de cinco y siete años, en tanto que estos no se diferencian entre sí.

\section{Rol de la edad en la regulación emocional}

Respecto de la regulación emocional, diversas investigaciones sugieren que dichas habilidades emergen de manera gradual a lo largo del tiempo. La maduración fisiológica, neurológica, física y cognitiva permite el desarrollo de un repertorio creciente de habilidades de regulación emocional (Thompson \& Goodvin, 2007). Particularmente, los avances en el lenguaje y la cognición mejoran la habilidad para hablar sobre los sentimientos, evitar conflictos a través de la negociación y emplear estrategias autorregulatorias con un mayor componente cognitivo.

Hipótesis 10. La regulación emocional (RE, positiva y negativa) se incrementa con la edad; los niños de tres años manifiestan niveles menores que los de cinco años, y estos menos que los de siete años.

\section{Rol del género en la atención focalizada, el control inhibi- torio, la emocionalidad y la regulación emocional}

En cuanto al rol del género en las variables de temperamento, vale resaltar algunos aportes del meta-análisis conducido por Else-Quest, Hyde, Goldsmith, y Van Hulle (2006). La magnitud de las diferencias de género en control voluntario fue notable; las niñas presentaban puntuaciones más elevadas. Específicamente, en control inhibitorio se apreció una diferencia moderada, mientras que en atención focalizada la diferencia fue pequeña. Con respecto a la afectividad negativa, el meta-análisis indicó que las diferencias de género no son significativas en el factor general ni en la mayoría de las dimensiones particulares. En el factor saliencia-extraversión la magnitud de las diferencias de género fue estadísticamente significativa, los varones presentaron puntuaciones más altas. Particularmente interesante es la dimensión de sonrisa, la cual ha sido señalada como uno de los indicadores más relevantes del afecto positivo (Rothbart, 1981). En esta dimensión el tamaño del efecto fue muy pequeño (Else-Quest et al., 2006). Las diferencias de género en emocionalidad, según señalan algunos autores (Vaughan Sallquist et al., 2009), han respondido a concepciones estereotipadas de los adultos, siendo las niñas quienes presentan mayores niveles de emocionalidad, aunque no siempre se han encontrado diferencias (Eisenberg et al., 2003; Rydell, Berlin \& Bohlin, 2003).

Hipótesis 11. Las niñas presentan una capacidad mayor de atención focalizada y control inhibitorio en relación con los varones.

Hipótesis 12. Las niñas y los varones no se diferencian en cuanto a la emocionalidad negativa.

Hipótesis 13. Existe diferencia de género en emocionalidad positiva/exuberancia, aunque no está clara la direccionalidad de la misma. 
De manera semejante, Cummings, Braungart-Rieker y Du Rocher-Schudlich (2003) señalan que son escasos los estudios que reportan diferencias de género en regulación emocional; al parecer, niñas y varones muestran una capacidad semejante para regular sus emociones (Rydell et al., 2003).

Hipótesis 14. Las niñas y los varones no se diferencian en la capacidad de regulación emocional (negativa y positiva).

Debido al rol predominante de los factores genéticos y biológicos en los rasgos temperamentales, las investigaciones que han indagado la incidencia del NSE son escasas, y los estudios que abordaron tal aspecto señalan efectos mínimos o directamente un efecto nulo (p.e., Garner \& Spear, 2000). En función de ello, no se plantearon hipótesis sobre diferencias en función del NSE en las distintas dimensiones del temperamento y la regulación emocional.

\section{MÉTODO}

\section{Participantes}

La selección de los participantes se realizó de manera no aleatoria. Participaron los padres y docentes de 623 niños de tres $(33.39 \%$, $45.19 \%$ niñas $)$, cinco $(33.87 \%$, $51.66 \%$ niñas) y siete años de edad $(32.74 \%, 53.92 \%$ niñas). Los niños asistían a instituciones públicas y privadas de Córdoba, Argentina. El nivel socio-económico (NSE) fue determinado a partir de un cuestionario sobre aspectos socio-demográficos que completaron los padres. Teniendo en cuenta la frecuencia observada en algunas de las siete categorías de NSE, las mismas se agruparon de la siguiente manera: NSE1 = marginal, bajo inferior y bajo superior; NSE2 = medio bajo; NSE3 = medio y medio alto; y NSE4 = alto. La participación de padres y docentes fue voluntaria, bajo condiciones de anonimato y confidencialidad.

\section{Instrumentos}

Cuestionario de datos socio-demográficos. A través de un cuestionario estructurado aplicado a los padres se recopiló información sobre distintos aspectos referidos al NSE: relación de aportantes y miembros del hogar, nivel educativo, ocupación y cobertura de salud del principal sostén del hogar, e indicadores de indigencia (Comisión de Enlace Institucional, AAM-SAIMO-CEIM, 2006).

Escalas de Comportamiento Preescolar y Jardín Infantil. Se empleó la versión abreviada de las escalas originalmente desarrolladas por Merrell (2003) producto de estudios psicométricos previos (Reyna \& Brussino, 2009a). Este instrumento permite evaluar habilidades sociales y problemas de conducta en niños de tres a siete años a través del informe de los docentes. La valoración del comportamiento del niño se realiza con base en una escala tipo Likert de cuatro puntos. Los ítems se suman y arrojan una puntuación total y para cada dimensión. Los índices de consistencia interna (a) en este estudio fueron: escala total de habilidades sociales $=.93$, Cooperación $=$ .86 , Interacción $=.86$, Independencia $=.84$; escala total de problemas de conducta $=.95$, Externalizante $=.95$, Internalizante $=.86$.

Cuestionario sobre Conducta Infantil. Se utilizaron versiones reducidas de las escalas originales propuestas por Rothbart et al. (2001), producto de estudios psicométricos previos (Reyna \& Brussino, 2009b). Este cuestionario evalúa aspectos referidos al temperamento en niños de tres a siete años a través del informe de los padres. Consta de 15 escalas; sin embargo, en este estudio solo se emplearon dos de ellas en función de las variables de interés: atención focalizada y control inhibitorio. La conducta del niño se clasifica en una escala tipo Likert de siete puntos. Para cada escala se obtiene una puntuación tras sumar los puntajes en los ítems que esta comprende. Los índices de consistencia interna (a) en este trabajo fueron: atención focalizada $=.71$, y control inhibitorio $=.80$.

Cuestionario Emocional. Se empleó el cuestionario propuesto por Rydell et al. (2003), el cual evalúa emocionalidad y regulación emocional con respecto a cuatro emociones a través del informe de los padres. La emocionalidad se valora con respecto a la frecuencia e intensidad de las reacciones, mientras que la regulación de emociones se evalúa en relación con la capacidad auto-regulatoria del niño y la habilidad para regular emociones con ayuda de otros. La puntuación de cada ítem se realiza con base en una escala tipo Likert de cinco puntos. En este estudio se utilizaron las escalas referidas a enojo, tristeza y emociones positivas/exuberancia. Con el fin de obtener una versión del cuestionario adecuada a las particularidades de la muestra de este trabajo, se realizó la traducción inversa del mismo, y se condujo un estudio piloto para evaluar la adecuación cultural, claridad semántica y aspectos gramaticales. Luego se efectuaron análisis factoriales exploratorios para las escalas de emocionalidad y regulación emocional, obteniéndose en cada caso estructuras bidimensionales. Los índices de consistencia interna (a) fueron: .77 emocionalidad negativa, .74 emocionalidad positiva/exuberancia, .79 regulación de enojo (RE), y .69 regulación de emociones positivas/exuberancia. Para cada dimensión se obtuvo una puntuación tras sumar los puntajes de los ítems que comprende. 


\section{Procedimiento}

Se contactó a las autoridades escolares de instituciones donde asistían niños de las edades de interés, y se invitó a los padres a participar en el estudio. Los padres fueron convocados a la institución en grupos reducidos para completar los cuestionarios sobre aspectos socio-demográficos, temperamento y emociones. A su vez, los docentes brindaron información sobre el comportamiento social de los niños autorizados a participar.

La recolección de datos fue realizada por la primera autora con la colaboración de asistentes de investigación. Los datos fueron analizados con el programa estadístico SPSS 19.

\section{RESULTADOS}

En primer lugar, se realizó una inspección inicial de los casos y de las variables comprendidas en este estudio con el fin de identificar las características que pudieran afectar el cumplimiento de los supuestos en los análisis posteriores.
Ninguna variable presentó más del 5\% de datos ausentes. La inspección inicial de la distribución de las variables mostró índices de asimetría y curtosis mayores a un valor aceptable ( \pm 1.5 , George \& Mallery, 2001) para algunas variables, tanto al considerar la muestra total como los distintos grupos etarios. Después se examinó la presencia de casos atípicos uni y multivariados, discriminando por grupos etarios. Se apreciaron 38 casos atípicos univariados $(Z> \pm 3)$ y 12 casos atípicos multivariados $(p<.001)$ (Tabachnick \& Fidell, 2001). En total, 46 niños mostraron atipicidad, 13 niños de tres años, 15 de cinco años y 18 de siete años. Posteriormente, se evaluó nuevamente la distribución de las variables excluyendo dichos casos; se observó que los índices de asimetría mejoraban, por lo cual se decidió excluir esos 46 casos de los análisis posteriores. Debido a ello, la muestra para los siguientes análisis quedó conformada por 577 niños. La Tabla 1 muestra los estadísticos descriptivos para cada una de las variables en la muestra total y distinguiendo por grupo etario.

Tabla 1.

Estadísticos descriptivos para la muestra total y cada grupo etario (tres, cinco y siete años)

\begin{tabular}{lcccccccc}
\hline & \multicolumn{2}{c}{$\begin{array}{c}\text { Total } \\
(\mathrm{N}=577)\end{array}$} & \multicolumn{2}{c}{$\begin{array}{c}3 \text { años } \\
(\mathrm{n}=195)\end{array}$} & \multicolumn{2}{c}{$\begin{array}{c}5 \text { años } \\
(\mathrm{n}=196)\end{array}$} & \multicolumn{2}{c}{$\begin{array}{c}7 \text { años } \\
(\mathrm{n}=186)\end{array}$} \\
\cline { 2 - 9 } HS & $\mathrm{M}$ & $\mathrm{DS}$ & $\mathrm{M}$ & $\mathrm{DS}$ & $\mathrm{M}$ & $\mathrm{DS}$ & $\mathrm{M}$ & $\mathrm{DS}$ \\
Coop & 43.54 & 8.95 & 34.70 & 5.71 & 48.36 & 6.59 & 47.73 & 6.68 \\
Inter & 16.66 & 3.44 & 13.40 & 2.32 & 18.70 & 2.43 & 17.91 & 2.78 \\
Indep & 17.88 & 3.94 & 14.89 & 2.67 & 19.21 & 3.86 & 19.60 & 3.31 \\
PC & 9.01 & 2.60 & 6.40 & 2.09 & 10.44 & 1.72 & 10.22 & 1.61 \\
Pe & 19.67 & 15.36 & 30.84 & 15.31 & 11.01 & 10.49 & 17.11 & 12.46 \\
Pi & 10.94 & 9.76 & 17.67 & 9.43 & 5.86 & 7.16 & 9.24 & 8.45 \\
AF & 8.73 & 6.30 & 13.17 & 6.85 & 5.15 & 4.04 & 7.87 & 4.68 \\
CI & 18.68 & 5.67 & 16.49 & 5.05 & 19.90 & 5.38 & 19.69 & 5.94 \\
EN & 33.37 & 8.60 & 30.15 & 6.74 & 34.94 & 8.66 & 35.08 & 9.32 \\
EPE & 25.19 & 7.67 & 31.39 & 4.57 & 20.84 & 6.19 & 23.28 & 7.53 \\
REN & 18.12 & 1.94 & 17.45 & 2.17 & 18.38 & 1.73 & 18.55 & 1.69 \\
REPE & 44.96 & 12.38 & 34.16 & 10.80 & 50.66 & 7.86 & 50.26 & 10.20 \\
\hline & 21.58 & 7.27 & 14.22 & 4.87 & 24.91 & 5.40 & 25.78 & 4.72 \\
\hline
\end{tabular}

Nota . HS = habilidades sociales; Coop = cooperación; Inter = interacción; Indep = independencia; $\mathrm{PC}=$ problemas de conducta; $\mathrm{Pe}=$ problemas externalizantes; $\mathrm{Pi}=$ problemas internalizantes; $\mathrm{AF}=$ atención focalizada $\mathrm{CI}=$ control inhibitorio; $\mathrm{EN}$ = emocionalidad negativa; $\mathrm{EPE}$ = emocionalidad positiva/exuberancia; $\mathrm{REN}$ = regulación de emociones negativas; REPE = regulación de emociones positivas/exuberancia. 
En segundo lugar, se desarrolló un análisis multivariado de la varianza (MANOVA) como una forma de controlar el porcentaje de error experimental producto de considerar numerosas variables dependientes. Concretamente, se realizó un MANOVA a tres vías de clasificación: edad (tres, cinco y siete años), género (varones y niñas), y NSE (NSE1, NSE2, NSE3 y NSE4). Las variables dependientes fueron las variables comportamentales (subescalas), las distintas facetas del temperamento analizadas y la emocionalidad y regulación emocional correspondiente a emociones positivas y negativas. Se estableció un nivel de significación de 05 . Los resultados indicaron efectos principales significativos con respecto a edad $[1$ de Wilks $=0.151, F(28,1080)=$ $\left.60.588, p<.000, \mathrm{~h}_{\mathrm{p}}{ }^{2}=.61\right]$ y género [1 de Wilks $=0.865$, $\left.F(14,540)=6.021, p<.000, \mathrm{~h}_{\mathrm{p}}{ }^{2}=.14\right]$. Además, la interacción entre edad y género resultó ser significativa $[1$ de Wilks $\left.=0.800, F(28,1080)=4.565, p<.000, \mathrm{~h}_{\mathrm{p}}{ }^{2}=.11\right]$.

En tercer lugar, se condujeron análisis de varianza (ANOVA) a tres vías de clasificación (edad, género y NSE) sobre cada una de las variables dependientes. Las comparaciones a posteriori se realizaron con el estadístico de Tukey. En todos los casos se estableció un nivel de significación de .05. A continuación se describen los resultados:

\section{Comportamiento social}

Al evaluar las diferencias entre los grupos en el comportamiento social se observaron efectos principales significativos de edad y género en todas las variables comportamentales, y de NSE en Interacción Social (véase Tabla 2). A partir de la inspección del $\mathrm{h}_{\mathrm{p}}^{2}$ se aprecia que el factor edad explicó altos porcentajes de la varianza parcial de las variables dependientes (24\% a $46 \%$ ); por otro lado, el aporte del factor género fue pequeño ( $2 \%$ a $5 \%$ ), y aún más reducido fue el aporte del NSE ( $0 \%$ a $2 \%)$.

Análisis a posteriori permitieron apreciar un apoyo parcial a las hipótesis propuestas respecto del efecto de la edad. Los niños de tres años presentaron un nivel menor de habilidades sociales (puntuación total y dimensiones) en relación con los niños de cinco y siete años, apoyando la Hipótesis 1; sin embargo, los niños de cinco y siete años solo se diferenciaron al considerar la Cooperación Social; los niños de cinco años mostraron niveles más altos, lo cual contradice dicha hipótesis. Con respecto a los problemas de conducta (puntuación total y dimensiones) se observó que los tres grupos etarios se diferenciaban entre sí: los niños de tres años mostraron las puntuaciones más elevadas, seguidas por los niños de siete y luego por los de cinco años. Este resultado apoya parcialmente las Hipótesis 2, en tanto que contradice la hipótesis de un incremento de problemas internalizantes con el pasar de los años (Hipótesis 3).
La direccionalidad de las diferencias debida al factor género se manifestó de la manera esperada (Hipótesis 4 y 5). Se observó que las niñas presentaban niveles más elevados de HS y los varones de problemas de conducta (puntuaciones totales y por dimensiones).

Por otra parte, la diferencia en Interacción Social según el NSE se evidenció entre los NSE3 en relación con los NSE2 y NSE4, en donde el primero presentó valores más elevados. Estos resultados no apoyan el efecto negativo de condiciones socio-económicas adversas sobre el comportamiento social, tal como se había hipotetizado (Hipótesis 6).

Además, resultaron significativas las interacciones entre edad y género para la puntuación total de habilidades sociales y las dimensiones de Cooperación e Interacción Social, y para la puntuación total de problemas de conducta y la dimensión de problemas externalizantes. El tamaño del efecto osciló entre $1 \%$ y $5 \%$. Comparaciones a posteriori permitieron apreciar, en general, evidencia a favor de las hipótesis de interacción planteadas (Hipótesis 4 y 5). Los niños de tres años presentaron menos habilidades sociales y más problemas de conducta (puntuaciones totales y dimensiones en las que se evidenció un efecto significativo) en relación con los otros grupos, sin distinción de género. En el otro extremo, las niñas de cinco años mostraron las puntuaciones más altas de habilidades sociales y más bajas de problemas de conducta (puntuación total y dimensiones), excepto en la dimensión Interacción Social, respecto de la cual se observó que tanto las niñas de cinco como las de siete años presentaban un mejor desempeño, diferenciándose estadísticamente del resto. En posiciones intermedias se encontraron los varones de cinco años y los niños de siete años (niñas y varones), con características particulares según la dimensión. Estos grupos intermedios no se diferenciaron entre sí al considerar la Cooperación Social; mientras que los varones de cinco años presentaron un nivel menor de Interacción Social en relación con los niños de siete años. Al considerar la puntuación total de habilidades sociales, los varones de cinco años mostraron un menor desempeño en relación con las niñas de cinco años; los varones de siete años no se distinguieron de ninguno de estos grupos. En cuanto a los problemas de conducta y problemas externalizantes, los varones de siete años mostraron puntuaciones más altas en relación con las niñas de la misma edad, en tanto que los varones de cinco años no se diferenciaron de ninguno de estos grupos.

Asimismo, se observó un efecto de interacción significativo de género y NSE sobre Independencia Social, aunque el aporte a la explicación de la varianza parcial de dicha variable fue pequeño (1\%). Comparaciones $a$ posteriori indicaron que los varones de NSE2 mostraban 
menos índices de Interacción Social en relación con las niñas de NSE1, NSE2 y NSE3; además, los varones de NSE3 tuvieron una puntuación menor respecto de las niñas del mismo NSE. El resto de las interacciones (dobles y triples) que comprendían al factor NSE no resultaron ser estadísticamente significativas.

\section{Atención focalizada y control inhibitorio}

El examen de las diferencias entre grupos en las dimensiones temperamentales "frías" comprendidas en este estudio reveló efectos principales significativos de edad tanto en atención focalizada como en control inhibitorio, y de género solo respecto del último (Tabla 3); la proporción de varianza parcial explicada osciló entre $1 \%$ y $6 \%$. Asimismo, resultó significativa la interacción de la edad y el género sobre ambas variables, con bajos valores $\mathrm{de}_{\mathrm{p}}{ }^{2}(.02)$.

Análisis a posteriori ofrecieron evidencia que apoyó parcialmente lo hipotetizado respecto del factor edad (Hipótesis 7). Los niños de tres años presentaron un nivel menor de atención focalizada y control inhibitorio respecto de los niños de cinco y siete años, aunque entre estos últimos no se apreciaron diferencias estadísticamente significativas. Respecto del factor género (Hipótesis 11), los resultados apoyaron la hipótesis planteada en cuanto al control inhibitorio: las niñas mostraron puntuaciones más elevadas en esta dimensión, mientras que no se apreciaron diferencias significativas en atención focalizada.
En análisis a posteriori referidos a los efectos de interacción de la edad y el género sobre la atención focalizada se observó que los varones de tres años mostraban puntuaciones estadísticamente menores al resto de los grupos, con excepción de las niñas de tres años. Estas últimas presentaron niveles menores solo respecto de las niñas de cinco años y los varones de siete años, no diferenciándose de los varones de cinco años ni de las niñas de siete años. Mientras que las niñas de siete años mostraron una puntuación menor de atención focalizada respecto de los varones de siete años. Entre los varones y niñas de cinco años, y los varones de siete años, no se apreciaron diferencias estadísticas significativas.

Con respecto al control inhibitorio, análisis a posteriori referidos a la incidencia de la interacción de la edad y el género indicaron que los niños de tres años presentaron las menores puntuaciones en relación con el resto de los grupos, a excepción de los varones de cinco años. Este último grupo, a su vez, presentó niveles menores de control inhibitorio respecto de los grupos restantes, con excepción de las niñas de siete años. Las niñas de cinco años y los niños de siete años no se diferenciaron entre sí en términos de significación estadística.

Por último, cabe señalar que el efecto principal debido al NSE y las interacciones dobles (Edad X NSE, Género X NSE), y la triple, que involucraba al NSE (Edad X Género X NSE) no resultaron estadísticamente significativos.

Tabla 2.

Resultados de los análisis de varianza para las variables de comportamiento social según edad, género y NSE

\begin{tabular}{|c|c|c|c|c|c|c|c|c|}
\hline & & HS & Coop & Inter & Indep & PC & $\mathrm{Pe}$ & $\mathrm{Pi}$ \\
\hline \multirow{2}{*}{ Edad } & $\mathrm{F}(2,553)$ & $230.59^{* * *}$ & $194.89^{* * *}$ & $95.92^{* * *}$ & $223.97^{* * *}$ & $100.75^{* * *}$ & $84.99^{* * *}$ & $94.37^{* * *}$ \\
\hline & hp2 & .46 & .41 & .26 & .45 & .27 & .24 & .25 \\
\hline \multirow{2}{*}{ Género } & $\mathrm{F}(1,553)$ & $30.84^{* * *}$ & $15.60^{* * *}$ & $30.44^{* * *}$ & $12.86^{* * *}$ & $24.68^{* * *}$ & $19.37^{* * *}$ & $25.55^{* * *}$ \\
\hline & hp2 & .05 & .03 & .05 & .02 & .04 & .03 & .04 \\
\hline \multirow{2}{*}{ NSE } & $F(3,553)$ & 2.39 & 0.78 & $2.90^{*}$ & 1.94 & 1.09 & 1.37 & 0.47 \\
\hline & hp2 & .01 & 0 & .02 & .01 & .01 & .01 & 0 \\
\hline \multirow{2}{*}{ Edad X Género } & $\mathrm{F}(2,553)$ & $9.89^{* * *}$ & $5.2^{* *}$ & $13.59^{* * *}$ & 1.16 & $3.8^{*}$ & $5.8^{* *}$ & 2.5 \\
\hline & hp2 & .03 & .02 & .05 & 0 & .01 & .02 & .01 \\
\hline \multirow{2}{*}{ Edad X NSE } & $F(6,553)$ & 0.56 & 0.96 & 0.57 & 0.42 & 0.59 & 0.4 & 1.16 \\
\hline & hp2 & .01 & .01 & .01 & 0 & .01 & 0 & .01 \\
\hline \multirow{2}{*}{ Género X NSE } & $\mathrm{F}(3,553)$ & 1.97 & 1.6 & 0.93 & $2.77^{*}$ & 2.13 & 2.39 & 1.42 \\
\hline & hp2 & .01 & .01 & 0 & .01 & .01 & .01 & .01 \\
\hline \multirow{2}{*}{$\begin{array}{l}\text { Edad X Género } \\
\text { X NSE }\end{array}$} & $F(6,553)$ & 1 & 0.26 & 1.57 & 0.64 & 0.92 & 0.99 & 1.05 \\
\hline & hp2 & .01 & 0 & .02 & .01 & .01 & .01 & .01 \\
\hline
\end{tabular}

Nota . HS = habilidades sociales; Coop = cooperación; Inter = interacción; Indep = independencia; $\mathrm{PC}=$ problemas de conducta; $\mathrm{Pe}=$ problemas externalizantes; $\mathrm{Pi}=$ problemas internalizantes; $\mathrm{NSE}=$ nivel socio-económico. ${ }^{*} p<.05 .{ }^{* *} p<.01 .{ }^{* * *} p<.001$. 
Emocionalidad y regulación emocional

Las comparaciones entre las medias de los grupos en las dimensiones de procesos emocionales evidenciaron efectos significativos debido a los factores edad y género (Tabla 3 ). Las proporciones de varianza parcial explicada debido a la edad (.06 a .46) fueron mayores que para el género (.01 a $.02)$. Asimismo, resultaron significativas las interacciones entre edad y género, con tamaños del efecto $\left(\mathrm{h}_{\mathrm{p}}^{2}\right)$ entre .01 y.02. No se observaron efectos principales debido al NSE; solo mostró significación estadística la interacción entre género y NSE sobre la emocionalidad negativa.

Análisis a posteriori permitieron identificar las diferencias entre los grupos. Con respecto al factor edad, los resultados apoyaron parcialmente lo hipotetizado (Hipótesis 8-10). Los tres grupos etarios se diferenciaron al considerar la emocionalidad negativa: los niños de tres años presentaron niveles mayores, seguidos por los niños de siete años, y finalmente por los niños de cinco años. Por el contrario, los niños de tres años presentaron los menores niveles en Emocionalidad Positiva/Exuberancia, como también de regulación de emociones negativas y positivas/exuberancia; mientras que los niños de cinco y siete años solo se diferenciaron en regulación de emociones positivas/exuberancia, los niños de cinco años evidenciaron niveles menores en esta variable.

En cuanto al factor género, en general, los resultados no apoyaron las hipótesis propuestas. Los varones mostraron puntajes más altos de emocionalidad positiva/exuberancia (Hipótesis 13), y también, contrario a lo esperado, de emocionalidad negativa (Hipótesis 12). A su vez, los varones presentaron niveles más bajos de regulación (de emociones negativa y de positivas/exuberancia) con respecto a las niñas, ofreciendo evidencia contraria a lo supuesto (Hipótesis14).

Al examinar los efectos de interacción entre edad y género que se evidenciaron en regulación emocional, se observó que con respecto a regulación de emociones negativas los niños de tres años presentaban puntuaciones significativamente menores que el resto de los grupos, mientras que los varones de cinco años puntuaron menos que las niñas de la misma edad, no diferenciándose de los niños de siete años. A su vez, las niñas de cinco años no se diferenciaron de los niños de siete años. Un patrón semejante se apreció en regulación de emociones positivas/exuberancia: los niños de tres años presentaron las menores puntuaciones, seguidos por el grupo de varones de cinco años y, finalmente, por las niñas de cinco años y las niñas y varones de siete años (estos tres últimos grupos no se distinguieron entre sí).

La inspección del efecto de interacción entre género y NSE sobre emocionalidad negativa indicó que las niñas del NSE2 presentaban un nivel menor en esta variable respecto de los varones de NSE1, NSE2 y NSE3. El resto de las interacciones que comprendía al NSE no resultaron significativas en términos estadísticos.

Tabla 3.

Resultados de los análisis de varianza para las variables de temperamento y regulación emocional según edad, género y NSE

\begin{tabular}{|c|c|c|c|c|c|c|c|}
\hline & & $\mathrm{AF}$ & $\mathrm{CI}$ & $\mathrm{EN}$ & EPE & REN & REPE \\
\hline \multirow{2}{*}{ Edad } & $F(2,553)$ & $15.96^{* * *}$ & $18.85^{* * *}$ & $112.55^{* * *}$ & $16.08^{* * *}$ & $152.37^{* * *}$ & $236.71^{* * *}$ \\
\hline & hp2 & .06 & .06 & .29 & .06 & .36 & .46 \\
\hline \multirow{2}{*}{ Género } & $\mathrm{F}(1,553)$ & 0.03 & $4.81^{*}$ & $9.77^{* *}$ & $6.58^{*}$ & $6.57^{*}$ & $10.92^{* *}$ \\
\hline & hp2 & 0 & .01 & .02 & .01 & .01 & .02 \\
\hline \multirow{2}{*}{ NSE } & $\mathrm{F}(3,553)$ & 1.01 & 2.05 & 0.14 & 0.18 & 0.61 & 2.01 \\
\hline & hp2 & .01 & .01 & 0 & 0 & 0 & .01 \\
\hline \multirow{2}{*}{ Edad X Género } & $\mathrm{F}(2,553)$ & $4.65^{* *}$ & $6.12^{* *}$ & 2.41 & 2.98 & $3.64^{*}$ & $6.22^{* *}$ \\
\hline & hp2 & .02 & .02 & .01 & .01 & .01 & .02 \\
\hline \multirow{2}{*}{ Edad X NSE } & $\mathrm{F}(6,553)$ & 0.56 & 0.99 & 0.62 & 1.14 & 1.37 & 0.5 \\
\hline & hp2 & .01 & .01 & .01 & .01 & .01 & .01 \\
\hline \multirow{2}{*}{ Género X NSE } & $\mathrm{F}(3,553)$ & 1.25 & 1.02 & $2.88^{*}$ & 0.66 & 1.61 & 1.2 \\
\hline & hp2 & .01 & .01 & .02 & 0 & .01 & .01 \\
\hline \multirow{2}{*}{ Edad X Género X NSE } & $F(6,553)$ & 0.88 & 0.63 & 1.11 & 1.23 & 1.32 & 0.17 \\
\hline & hp2 & .01 & .01 & .01 & .01 & .01 & 0 \\
\hline
\end{tabular}

Nota $. \mathrm{AF}=$ atención focalizada; $\mathrm{CI}=$ control inhibitorio; $\mathrm{EN}=$ emocionalidad negativa; $\mathrm{EPE}=$ emocionalidad positiva/exuberancia; $\mathrm{REN}=$ regulación de emociones negativas; REPE = regulación de emociones positivas/exuberancia; NSE = nivel socio-económico. ${ }^{*} p<.05 .{ }^{* *} p<.01 .{ }^{* * *} p<.001$. 


\section{DISCUSIÓN}

El objetivo de este trabajo fue evaluar las diferencias en función de la edad (tres, cinco y siete años), género (masculino y femenino), y nivel socio-económico (NSE1, NSE2, NSE3, NSE4) en habilidades sociales y problemas de conducta, atención focalizada, control inhibitorio, emocionalidad (positiva y negativa) y regulación emocional (positiva y negativa) en una muestra de niños cordobeses (Argentina).

Con respecto al comportamiento social, se observaron diferencias principalmente según la edad y el género de los niños, explicando el primero altos porcentajes de la varianza parcial de las variables dependientes, y bastante menores el segundo; mientras que solo se apreciaron diferencias de NSE en la dimensión de Interacción Social, con un reducido aporte a la explicación de la varianza.

Las hipótesis propuestas referidas al factor edad recibieron un apoyo parcial. Los niños de tres años mostraron el menor nivel de habilidades sociales (puntuación total y dimensiones) y el mayor nivel de problemas de conducta (puntuación total y dimensiones), lo cual aportó evidencia a favor de las hipótesis sobre habilidades sociales, problemas de conducta y problemas externalizantes, pero contradijo la hipótesis de un mayor nivel de problemas internalizantes con el pasar de los años.

Por otra parte, las diferencias observadas debido al género de los niños estuvieron en la dirección esperada; las niñas manifestaron mejores habilidades sociales y los varones más PC (puntuaciones totales y dimensiones). Asimismo, recibieron apoyo parcial las hipótesis propuestas respecto de los efectos de interacción entre la edad y el género. En etapas tempranas no se apreciaron diferencias de género; todos los niños de tres años se caracterizaron por un peor comportamiento social. En cambio, se apreciaron algunas diferencias de género en etapas posteriores, aunque no siempre en la dirección esperada. En general, las niñas de cinco años fueron las que evidenciaron un mejor comportamiento social, con niveles más altos de habilidades sociales y más bajos de problemas de conducta, mientras que los grupos restantes (varones de cinco años y todos los niños de siete años) estuvieron en niveles intermedios.

En términos generales, las diferencias de género observadas están en línea con lo señalado por otros autores (Bandeira et al., 2006; Connor, 2002; Persson, 2005; Teague, 2005). En cambio, los resultados obtenidos respecto de la edad parecen un tanto contradictorios. Por una parte, ofrecen evidencia acerca de las escasas habilidades sociales y mayores problemas de conducta en edades tempranas (Miner \& Clarke-Stewart, 2008; Thompson \& Goodvin,
2007; Vahedi, Farrokhi \& Farajian, 2012). Sin embargo, también se encontró que los niños de menor edad manifiestan mayores problemas internalizantes, los cuales se supone requieren de un mayor desarrollo cognitivo y emocional (Kovacs \& Devline, 1998). Además, el mejoramiento en el desempeño social parece depender de factores tales como el género, ya que fueron las niñas de cinco años las que mostraron un mejor comportamiento social. Como se señalara en un estudio previo (Reyna, Ison \& Brussino, 2011), tal vez las diferencias entre los contextos preescolary escolar en los que están insertos los docentes que valoraron el comportamiento social de los niños haya afectado, al menos en parte, los resultados encontrados. De hecho, otros investigadores ya han subrayado la relevancia del contexto de valoración (Brophy-Herb, Lee, Nievar \& Stollak, 2007; Lacunza, 2014) y la importancia del informante considerado (Bandeira et al., 2006).

La hipótesis referida al impacto de condiciones socioeconómicas adversas sobre el comportamiento social no fue corroborada, hallándose en escasas ocasiones diferencias relacionadas con el NSE, con una direccionalidad no clara (p.e., interacción social). Este resultado contradice aquellos estudios que han señalado efectos negativos de un bajo nivel socio-económico (Bandeira et al., 2006; Propper \& Rigg, 2007), pero está en línea con lo observado por investigadores de otras latitudes (Turquía), quienes reportan un efecto no significativo del NSE del colegio, el estatus educativo y las características ocupacionales de los padres sobre las habilidades sociales de niños de escolaridad primaria (Saygili, 2014). Probablemente, uno de los factores que más haya incidido en la ausencia de diferencias sea la muestra utilizada; si bien se procuró que todos los NSE estuviesen representados, el muestreo fue no probabilístico accidental.

Las variables temperamentales "frías" que comprendió este trabajo fueron atención focalizada y control inhibitorio. Se apreciaron diferencias de edad en ambos procesos $\mathrm{y}$ de género solo en el último, aunque en todos los casos la proporción de varianza parcial explicada fue reducida; no se apreciaron efectos debidos al NSE.

Los niños de tres años, tal como se supuso, mostraron menos habilidades de atención focalizada y control inhibitorio en relación con el resto de los niños; sin embargo, no hubo diferencias significativas entre los niños de cinco y siete años, es decir que el desarrollo de tales habilidades entre los cinco y siete años no es linealmente creciente sino que adquiere la forma de una meseta. Por otro lado, las diferencias de género observadas estuvieron en la dirección esperada: aunque solo se evidenciaron en control inhibitorio, las niñas mostraron mejores habilidades en este comportamiento. Además, se apreciaron efectos de 
interacción entre la edad y el género, los cuales revelaron que los varones de tres años presentaron los niveles más bajos de atención focalizada, y los niños de cinco y los varones de siete años los niveles más altos. Mientras que todos los niños de tres años mostraron menores habilidades de control inhibitorio, las niñas de cinco años y los varones de siete fueron quienes presentaron los niveles más altos.

En general, los resultados obtenidos concuerdan con las diferencias evolutivas reportadas en otros estudios (Kochanska et al., 2000; Macdonald, Beauchamp, Crigan \& Anderson, 2014; Posner \& Rothbart, 2000; Rothbart, Ahadi, et al., 2001); y también con las diferencias de género en control inhibitorio a favor de las niñas que señala la revisión meta-analítica conducida por Else-Quest et al. (2006), aunque no se encontraron diferencias en atención focalizada como puso en evidencia tal revisión. Quizás, un análisis pormenorizado de los estudios comprendidos en el meta-análisis permitiría identificar las causas de tal discrepancia. En principio, se ponderan cuestiones de orden metodológico y cultural como los principales factores diferenciales.

Por último, se resaltan los principales hallazgos respecto de las diferencias en los procesos emocionales evaluados, emocionalidad y regulación emocional respecto de emociones positivas y negativas. Se observaron diferencias tanto debido a la edad como al género, pero no al considerar el NSE. La edad explicó mayores porcentajes de varianza parcial que el factor género.

Con respecto a la edad, los niños de tres años mostraron niveles más altos de emocionalidad negativa; sin embargo, en posición intermedia estuvieron los niños de siete años y no los de cinco años como se hipotetizaba. Mientras que en emocionalidad positiva/exuberancia, contrario a lo supuesto, los niños de tres años presentaron las puntuaciones más bajas, los de cinco y siete años, como se hipotetizó, no se diferenciaron entre sí. Por otra parte, las diferencias en regulación emocional apoyaron, en general, la hipótesis de un incremento de la capacidad regulatoria con el pasar de los años, aunque los niños de cinco y siete años no se diferenciaron en la regulación de emociones positivas/ exuberancia.

De esta manera, se aprecia que las diferencias evolutivas observadas en emocionalidad negativa coinciden con los altos niveles señalados por otros investigadores en edades tempranas (Murphy et al., 1999). No obstante, el incremento parece no ser lineal, lo cual probablemente se relacione con la semejanza en regulación de emociones negativas en niños de cinco y siete años, quienes manifestaron una mayor capacidad regulatoria en relación con los niños de tres años. Por ello, los resultados solo resultan parcialmente coincidentes con los estudios que manifiestan un incremento de la regulación emocional con los años (Thompson \& Goodvin, 2007). Además, estos resultados tal vez estén reflejando la existencia de un efecto de interacción entre emocionalidad y regulación emocional, como indican algunos investigadores (p.e., Eisenberg, Cumberland, et al., 2001). Por otra parte, la divergencia entre los hallazgos que indican un incremento de emocionalidad positiva/exuberancia a partir de los tres años (Guerin \& Gottfried, 1994) y lo observado en este estudio se atribuye, principalmente, a diferencias en las medidas de evaluación utilizadas; sin embargo, debido al escaso conocimiento que revela la literatura respecto de esta dimensión, cualquier conclusión tendría un elevado margen de error.

En cuanto al género, los varones presentaron niveles más altos de emocionalidad negativa, lo cual contradice la igualdad de género supuesta; $y$ también mostraron niveles más altos de emocionalidad positiva/exuberancia, respondiendo así el interrogante de direccionalidad planteado. Por otro lado, los varones mostraron una menor capacidad regulatoria tanto respecto de emociones positivas como negativas, contrario a la semejanza que se hipotetizó. Cabe señalar que también se apreciaron efectos de interacción en la regulación de emociones. Los niños de tres años presentaron el nivel más bajo de regulación de emociones negativas y positivas/exuberancia, mientras que los varones de cinco años estuvieron en un nivel intermedio; el grupo de varones de siete años y las niñas de cinco años se caracterizaron por manifestar mejores habilidades para regular emociones.

Estos resultados se oponen a la semejanza en emocionalidad negativa observada por Else-Quest et al. (2006) y también en el estudio de Rydell et al. (2003). En cambio, suponiendo cierta semejanza entre la dimensión de emocionalidad positiva/exuberancia utilizada en este estudio y el factor de saliencia-extraversión del modelo de Rothbart (1981), los resultados coinciden con el menor nivel que manifiestan las niñas en dicho factor según el estudio metaanalítico de Else-Quest et al. Finalmente, las diferencias en Regulación Emocional a favor de las niñas contradicen la ausencia de efecto del factor género señalada por otros investigadores (Cummings et al., 2003; Rydell et al., 2003). Es probable que las divergencias señaladas estén vinculadas con una asociación marcada entre los procesos emocionales y el comportamiento social en la muestra analizada (los varones mostraron un peor desempeño), aunque solo futuros estudios podrán esclarecer este punto.

En síntesis, el factor más determinante en las diferencias fue la edad, según lo cual los niños de tres años manifestaron un peor desempeño en relación con los niños de mayor 
edad al considerar las distintas variables. No obstante, el desarrollo parece no ser lineal y evolutivo en todos los procesos en el período etario comprendido, ya que los niños de cinco años mostraron en algunos casos un desempeño semejante o mejor en relación con los niños de siete años. En cuanto al género, las niñas mostraron contar con mejores habilidades en todos los procesos bajo análisis; sin embargo, esas diferencias resultaron aparentes sobre todo a partir de los cinco años. Por último, el NSE no impactó de manera significativa las variables analizadas en este trabajo.

\section{REFERENCIAS}

Achenbach, T.M. (1966). The classification of children's psychiatric cymptoms: A factor-analytic study. Psychological Monographs, 80(7), 1-37.

Bandeira, M., Silva Rocha, S., Cordeiro Freitas, L., Pereira Del Prette, Z.A. \& Del Prette, A. (2006). Habilidades sociais e variáveis sociodemográficas em estudantes do ensino fundamental. Psicologia em Estudo, 11(3), 541-549.

Bradley, R.H. \& Corwyn, R.F. (2002). Socioeconomic status and child development. Annual Review of Psychology, 53, 371-399.

Bridges, L.J., Denham, S.A. \& Ganiban, J.M. (2004). Definitional issues in emotion regulation research. Child Development, 75(2), 340-345.

Brooks-Gunn, J. \& Duncan, G.J. (1997). The effects of poverty on children. The Future of Children, 7(2), 55-71.

Brophy-Herb, H. E., Lee, R. E., Nievar, M. A. \& Stollak, G. (2007). Preschoolers' social competence: Relations to family characteristics, teacher behaviors and classroom climate. Journal of Applied Developmental Psychology, 28(2), 134-148.

Cicchetti, D. \& Toth, S.L. (1991). A developmental perspective on internalizing and externalizing disorders of childhood. In D. Cicchetti \& S. L. Toth (Eds.), Rochester Symposium on Developmental Psychopathology: Internalizing and externalizing expressions of dysfunction (Vol. 2, pp. 1-19). Rochester, NY: University of Rochester Press.

Cole, P.M., Martin, S.E. \& Dennis, T. (2004). Emotion regulation as a scientific construct: Methodological challenges and directions for child development research. Child Development, 75(2), 317-333.

Comisión de Enlace Institucional, AAM-SAIMO-CEIM (2006). Nivel socio-económico 2006. Recuperado en diciembre de 2008 de http://www.saimo.org.ar/socios/ Socios/NSE200623nov2006-Informe_final.pdf

Connor, D.F. (2002). Aggression and antisocial behavior in children and adolescents. New York: The Guilford Press.

Cummings, E.M., Braungart-Rieker, J. \& Du Rocher Schudlich, T. (2003). Emotion and personality development in childhood. In I. B. Weiner (Ed.), R. M. Lerner, M. A. Easterbrooks \& J. Mistry (Vol. Eds.), Comprehensive handbook of Psychology,
Vol. 6: Developmental Psychology (pp.211-239). New York: John Wiley \& Sons, Inc.

Diamond, L.M. \& Aspinwall, L.G. (2003). Emotion regulation across the life span: An integrative perspective emphasizing self-regulation, positive affect, and dyadic processes. Motivation \& Emotion, 27(2), 125-156.

Eisenberg, N. \& Spinrad, T.L. (2004). Emotion-related regulation: Sharpening the definition. Child Development, 75(2), 334-339.

Eisenberg, N., Cumberland, A., Spinrad, T.L., Fabes, R.A., Shepard, S.A. \& Reiser, M. (2001). The relations of regulation and emotionality to children's externalizing and internalizing problem behavior. Child Development, 72(4), 1112-1134.

Eisenberg, N., Fabes, R.A. \& Guthrie, I.K. (1997). Coping with stress. The roles of regulation and development. In S. A. Wolchik \& L. N. Sandler (Eds.), Handbook of children's coping: Linking theory and intervention (pp. 41-70). New York: Plenum.

Eisenberg, N., Fabes, R.A., Guthrie, I.K. \& Reiser, M. (2000). Dispositional emotionality and regulation: Their role in predicting quality of social functioning. Journal of Personality \& Social Psychology, 78(1), 136-157.

Eisenberg, N., Fabes, R.A., Guthrie, I.K., Murphy, B.C., Maszk, P., Holmgren, R. \& Suh, K. (1996). The relations of regulation and emotionality to problem behavior in elementary school children. Development and Psychopathology, 8(1), 141-162.

Eisenberg, N., Zhou, Q., Losoya, S.H., Fabes, R.A., Shepard, S.A., Murphy, B. C.,... Cumberland, A. (2003). The relations of parenting, effortful control, and ego control of children's emotional expressivity. Child Development, 74(3), 875-895.

Else-Quest, N.M., Hyde, J.S., Goldsmith, H.H. \& Van Hulle, C.A. (2006). Gender differences in temperament: A meta-analysis. Psychological Bulletin, 132(1), 33-72.

Garner, P.W. \& Spears, F.M. (2000). Emotion regulation in low-income preschoolers. Social Development, 9(2), 246-264.

Gartstein, M.A. \& Rothbart, M.K. (2003). Studying infant temperament via the Revised Infant Behavior Questionnaire. Infant Behavior and Development, 26(1), 64-86.

George, D. \& Mallery, M.P. (2001). SPSS for Windows step by step: A simple guide and reference. Boston, MA: Allyn \& Bacon.

Gottlieb, G. (2003). On making behavioral genetics truly developmental. Human Development, 46(6), 337-355.

Grantham-McGregor, S., Cheung, Y.B., Cueto, S., Glewwe, P., Richter, L. \& Strupp, B. (2007). Child development in developing countries 1: Developmental potential in the first 5 years for children in developing countries. Lancet, 369(9555), 60-70.

Gresham, F.M. (2001). Assessment of social skills in children and adolescents. In D. H. Saklofske, J. J. W. Andrews \& H. L. Janzen (Eds.), Handbook of Psychoeducational Assessment: A practical handbook (pp. 325-355). San Diego, CA: Academic Press. 
Gresham, F.M., Sugai, G. \& Horner, R.H. (2001). Interpreting outcomes of social skills training for students with highincidence disabilities. Exceptional Children, 67(3), 331-344.

Guerin, D.W. \& Gottfried, A.W. (1994). Developmental stability and change in parent reports of temperament: A ten-year longitudinal investigation from infancy through preadolescence. Merrill-Palmer Quarterly, 40(3), 334-355.

Hill, A., Degnan, K., Calkins, S.D. \& Keane, S.P. (2006). Profiles of externalizing behavior problems for boys and girls across preschool: The roles of emotion regulation and inattention. Developmental Psychology, 42(5), 913-928.

Holodynski, M. \& Friedlmeier, W. (2006). Development of emotions and emotion regulation. New York: Springer

Keenan, K. \& Shaw, D. (1997). Developmental and social influences on young girls' early problem behaviors. Psychological Bulletin, 121(1), 95-113.

Keiley, M.K., Lofthouse, N., Bates, J.E., Dodge, K.A. \& Pettit, G.S. (2003). Differential risks of covarying and pure components in mother and teacher reports of externalizing and internalizing behavior across ages 5 to 14. Journal of Abnormal Child Psychology, 31(3), 267-283.

Kochanska, G., Murray, K. \& Harlan, E.T. (2000). Effortful control in early childhood: Continuity and change, antecedents, and implications for social development. Development Psychology, 36(2), 220-232.

Kovacs, M. \& Devline, B. (1998). Internalizing disorders in childhood. Journal of Child Psychology and Psychiatry, 39(1), 47-63.

Lacunza, A. B. (2014). Social skills of children in vulnerable conditions in Northern Argentina. In A. Castro Solano (Ed.), Positive Psychology in Latin America (pp. 213-229). New York: Springer.

Macdonald, J. A., Beauchamp, M. H., Crigan, J. A. \& Anderson, P. J. (2014). Age-related differences in inhibitory control in the early school years. Child Neuropsychology: A Journal on Normal and Abnormal Development in Childhood and Adolescence, 20(5), 509-526.

Merrell, K.W. (2003). Preschool and Kindergarten Behavior Scales, Second Edition: Spanish Language Version. Austin, TX: PRO-ED.

Miner, J.L. \& Clarke-Stewart, K.A. (2008). Trajectories of externalizing behavior from age 2 to age 9: Relations with gender, temperament, ethnicity, parenting, and rater. Developmental Psychology, 44(3), 771-786.

Murphy, B.C., Eisenberg, N., Fabes, R.A., Shepard, S. \& Guthrie, I.K. (1999). Consistency and change in children's emotionality and regulation: A longitudinal study. Merrill-Palmer Quarterly, 45(3), 413-444.

Ostrov, J.M. \& Keating, C.F. (2004). Gender differences in preschool aggression during free play and structured interactions: An observational study. Social Development, 13(2), 255-277.
Persson, G.E. B. (2005). Developmental perspectives on prosocial and aggressive motives in preschoolers' peer interactions. International Journal of Behavioral Development, 29(1), 80-91.

Posner, M.I. \& Rothbart, M.K. (2000). Developing mechanisms of self-regulation. Development and Psychopathology, 12(3), 427-441.

Propper, C. \& Rigg, J. (2007). Socio-cconomic status and child behaviour: Evidence from a contemporary UK cohort (CASE Paper 125). Recuperado de http://sticerd.lse. ac.uk/dps/case/ cp/CASEpaper125.pdf

Reyna, C.E. \& Brussino, S.A. (2009a). Propiedades psicométricas de la escala de comportamiento preescolar y jardín infantil en una muestra de niños argentinos de 3 a 7 años. Psykhe, 18(2), 127-140.

Reyna, C.E. \& Brussino, S.A. (2009b). Temperamento infantil: Estudio de fiabilidad de escalas del Cuestionario de Conducta Infantil de Rothbart. Libro del XIII Congreso Argentino de Psicología, Compromiso social frente a las problemáticas actuales (pp. 769-775). Córdoba: CPPC.

Reyna, C.E., Ison, M.S. \& Brussino, S.A. (2011). Comportamiento social y procesamiento de la información social en niños argentinos. International Journal of Psychology and Psychological Therapy, 11(1), 57-78.

Rose-Krasnor, L. (1997). The nature of social competence: A theoretical review. Social Development, 6(1), 111-135.

Rothbart, M.K. (1981). Measurement of temperament in infancy. Child Development, 52(2), 569-578.

Rothbart, M.K. \& Bates, J.E. (1998). Temperament. In W. Damon (Serie Ed.), N. Eisenberg (Vol. Ed.). Handbook of Child Psychology, Vol. 3: Social, emotional, and personality development (5th ed., pp.105-176). New York: Wiley.

Rothbart, M.K., Ahadi, S.A. \& Evans, D.E. (2000). Temperament and personality: Origins and outcomes. Journal of Personality \& Social Psychology, 78(1), 122-135.

Rothbart, M.K., Ahadi, S., Hershey, K.L. \& Fisher, P. (2001). Investigations of temperament at three to seven years: The Children's Behavior Questionnaire. Child Development, 72(5), 1394-1408.

Rothbart, M.K., Chew, K.H. \& Gartstein, M.A. (2001). Assessment of temperament in early development. In L.T. Singer \& P.S. Zeskind. (Eds.), Biobehavioral assessment of the infant (pp. 190-208). New York: Guildford Press.

Rydell, A.-M., Berlin, L. \& Bohlin, G. (2003). Emotionality, emotion regulation, and adaptation among 5- to 8-year-old children. Emotion, 3(1), 30-47.

Saygili, G. (2014). Social skills of children in public primary school in Turkey: A case study. International Journal of Recent Scientific Research, 5(1), 148-152.

Sewrud-Clikeman, M. (2007). Social competence in children. New York: Springer.

Sheridan, S.M. \& Walker, D. (1999). Social skills in context: Considerations for assessment, intervention, and generaliza- 
tion. In C.R. Reynolds \& T.B. Gutkin(Eds.), The Handbook of School Psychology (3rd ed., pp.686-708). New York: Wiley.

Shiner, R.L. \& Caspi, A. (2003). Personality differences in childhood and adolescence: Measurement, development, and consequences. Journal of Child Psychology and Psychiatry, 44(1), 2-32.

Souza, F. \& Rodrigues, M.M.P. (2002). A segregação sexual na interação de crianças de 8 e 9 anos. Psicologia: Reflexão e Crítica, 15(3), 489-496.

Tabachnick, B. \& Fidell, L. (2001). Using multivariate statistics (4th ed.). New York: Harper \& Row.

Teague, R.J.P. (2005). Social functioning in preschool children: Can social information processing and self-regulation skills explain sex differences and play a role in preventing ongoing problems? (Unpublished doctoral dissertation) Griffith University, Australia.
Thompson, R.A. \& Goodvin, R. (2007). Taming the tempest in the teapot: Emotion regulation in toddlers. In C. A. Brownell \& C. B. Kopp (Eds.), Transitions in early socioemotional development: The toddler years (pp.320-344). New York: Guilford.

Trianes, M.V., Muñoz, A.M. \& Jiménez, M. (1997). Competencia social: su educación y tratamiento. Madrid: Pirámide.

Vahedi, S., Farrokhi, F. \& Farajian, F. (2012). Social competence and behavior problems in preschool children. Iranian Journal of Psychiatry, 7(3), 126-134.

Vaughan Sallquist, J., Eisenberg. N., Spinrad, T.L., Reiser, M., Hofer, C., Liew, J.,... Eggum, N. (2009). Positive and negative emotionality: Trajectories across six years and relations with social competence. Emotion, 9(1), 15-28. 